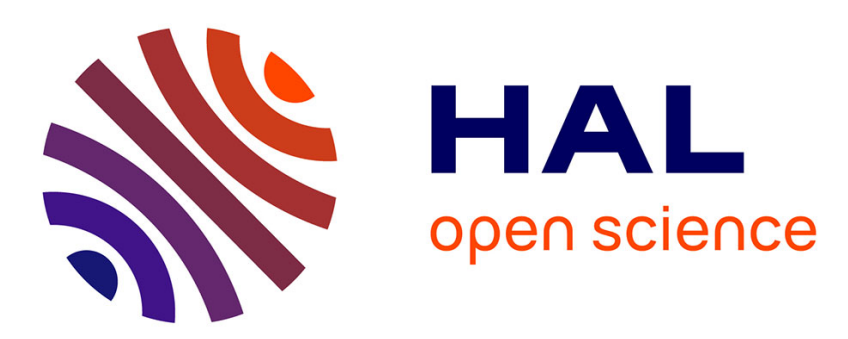

\title{
Ionization potential depression for non equilibrated aluminum plasmas
}

\author{
Annette Calisti, Sandrine Ferri, Bernard Talin
}

\section{To cite this version:}

Annette Calisti, Sandrine Ferri, Bernard Talin. Ionization potential depression for non equilibrated aluminum plasmas. Journal of Physics B: Atomic, Molecular and Optical Physics, 2015, 10.1088/09534075/48/22/224003. hal-01208087

\section{HAL Id: hal-01208087 \\ https://hal.science/hal-01208087}

Submitted on 15 Jan 2016

HAL is a multi-disciplinary open access archive for the deposit and dissemination of scientific research documents, whether they are published or not. The documents may come from teaching and research institutions in France or abroad, or from public or private research centers.
L'archive ouverte pluridisciplinaire HAL, est destinée au dépôt et à la diffusion de documents scientifiques de niveau recherche, publiés ou non, émanant des établissements d'enseignement et de recherche français ou étrangers, des laboratoires publics ou privés. 


\title{
Ionization potential depression for non equilibrated aluminum plasmas
}

\author{
A. Calisti, S. Ferri and B. Talin \\ Aix Marseille Universite, CNRS, PIIM UMR 7345, 13397, Marseille, France \\ E-mail: annette.calisti@univ-amu.fr
}

March 2015

\begin{abstract}
A classical Molecular Dynamics simulation model, designed to simulate neutral plasmas with various charge states of a given atom together with electrons, is used to investigate the ionization potential depression (IPD) in dense plasmas. The IPD is discussed for aluminum plasma at and out of equilibrium. The simulation results are compared with those of earlier theoretical models and with experimental data obtained in the framework of x-ray free-electron laser experiments. The model proposed in this work appears as an important tool to provide data for further discussion on IPD models.
\end{abstract}

PACS numbers: 52.65.Yy, 52.25.b, 52.27.Gr

Keywords: Ionization Potential Depression, Classical Molecular Dynamics Simulations, Multi Component Plasma Simulations

\section{Introduction}

The ionization potential depression (IPD) of an ion in a dense plasma is somehow an average quantity characterizing the global effect of the plasma on that ion. Quantum properties, e.g., the ionization potential of an ion are modified due to the interactions of the valence electron with the whole of the surrounding charges. Essentially two distinct theoretical models, the Stewart-Pyatt (SP) [1] and Ecker-Kröll (EK) [2] models have been developed and used to describe the IPD. Recently, their validity has been discussed in the framework of two experiments, one using an x-ray free-electron laser [3] and the other one using a high-power optical laser [4] to create the dense plasma. It seems that neither the SP model nor the EK model be able to explain both experiments. This has initiated a renewed interest for the problem of the ionization potential depression in dense plasmas, see for instance $[5,6,7,8,9]$.

In a previous study of the IPD [10], we considered an aluminum plasma in equilibrium, i.e. with equal ion and electron temperature. Such conditions are quite academic in regard of the true experimental process of generation of plasmas for IPD 
studies by impact of a short radiation pulse on a solid target $[11,3]$. In these experiments, the electrons within the target are heated, within $80 \mathrm{fs}$, to temperature between $70 \mathrm{eV}$ and $180 \mathrm{eV}$ depending on the photon energy of the irradiation. Moreover, the $K-$ shell fluorescence, on which the interpretation of the experiment is based, only occurs while the target is irradiated ( $80 \mathrm{fs}$ ). On this time scale, the ion motion is negligible and emission occurs in a plasma where the ion density $n_{i}$ is the solid-density and where the electron density is given by $n_{e}=\bar{Z} n_{i}$, with $\bar{Z}$ the ionization amount. To get closer of these conditions one can use MD to simulate a two component plasma of ions at room temperature and solid density, and electrons in pseudo equilibrium with the cold ion population. The argument here is that the electron adjustment to the ions can occur in a time that does not allow the ion population to be heated by the electrons. The code BINGO-TCP $[12,13]$ has been used to match approximately these conditions, with typically the ion temperature, $T_{i}=300 \mathrm{~K}$ and two electron temperatures, $T_{e}=50 \mathrm{eV}$ or $T_{e}=160 \mathrm{eV}$. The purpose of this work is the evaluation of the differences induced on the IPD by these two kinds of plasmas at and out of equilibrium.

The real question that arises here, is : does the generation process of the plasma can affect the IPD measurement?

\section{Model summary}

Both the theoretical and simulation models have been recalled elsewhere [10]. Their following mention is limited to a few guidelines.

\subsection{Theoretical models}

Two theoretical models formulated in the sixties for plasmas in equilibrium will be used in the following for discussions. These models are of importance as they are commonly used for dense plasma modeling.

The Stewart and Pyatt model [1] is based on the finite-temperature Thomas-Fermi potential for the average electrostatic potential near nuclei of the plasma particles. The bound electrons are considered as part of the unperturbed ion and do not contribute to the reduction of the ionization energy. The plasma free electrons are described by Fermi-Dirac statistics and ions by Maxwell-Boltzmann statistics. The SP model relates the IPD of an ion of charge $Z$ to the generalized Debye length $\lambda_{D}$ :

$$
\begin{aligned}
& \Delta U_{S P}(Z)=\frac{3 Z e^{2}}{2 r_{0}}\left\{\left[1+\left(\frac{\lambda_{D}}{r_{0}}\right)^{3}\right]^{2 / 3}-\left(\frac{\lambda_{D}}{r_{0}}\right)^{2}\right\} \\
& \lambda_{D}=\sqrt{\frac{k T}{4 \pi\left(n_{i}+n_{e}\right) e^{2}}}, \frac{4 \pi n_{i} r_{0}^{3}}{3}=1
\end{aligned}
$$

where $Z$ is the charge state of the atom (or ion) after the ionization occurrence $(Z=1$ for neutrals), $r_{0}$ the average inter-ionic distance for the average ion charge $\bar{Z} . n_{i}$ is 
the corresponding ion density and $n_{e}$ the electron density. In the high density or low temperature limit, the IPD becomes:

$$
\Delta U_{S P}(Z)=\frac{3 Z e^{2}}{2 r_{0}}
$$

Second the Ecker-Kröll model [2] reads

$$
\Delta U_{E K}(Z)=Z e^{2}\left\{\begin{array}{cc}
1 / \lambda_{D} & n_{c r} \geq n_{i}(1+\bar{Z}) \\
C \times(1+\bar{Z})^{1 / 3} / r_{0} & n_{c r}<n_{i}(1+\bar{Z})
\end{array}\right.
$$

where

$$
n_{c r}=\frac{3}{4 \pi}\left(\frac{k T}{Z_{N}^{2} e^{2}}\right)^{3}
$$

is the critical density which includes both ion and free electron densities, $Z_{N}$ being the nuclear charge $\left(Z_{N}=13\right.$ for $\left.\mathrm{Al}\right)$ and $T$ the plasma temperature. The constant $C$ is determined by imposing the continuity of the IPD at the critical density:

$$
C=\left(\frac{r_{0}}{(1+\bar{Z})^{1 / 3} \lambda_{D}}\right)_{n_{c r}}
$$

None of these two models allows ionic and electronic distinct temperature. In the following, we will compare our simulation results with both models SP and modified EK with $\mathrm{C}=1$ according to experimental considerations [3].

\subsection{Molecular dynamics, potentials and ionization / recombination protocol}

The BINGO-TCP simulation code described in $[12,13]$ involves two major specificities.

First, an ion / electron potential depends on the ion charge $Z_{i}$ through the characteristic length $\delta\left(Z_{i}\right)$

$$
V_{i e}(r)=-Z_{i} e^{2} e^{-r / \lambda}\left(1-e^{-r / \delta\left(Z_{i}\right)}\right) / r .
$$

This choice implies that an electron located at an ion $(r=0)$ occupies the fundamental state of the ion whose charge is $Z_{i}$ with a core charge $Z_{i}+1$ and with the expected ionization potential for that ion

$$
E_{i}=Z_{i} e^{2} / \delta\left(Z_{i}\right)
$$

The screening parameter $\lambda$ reminds that in our simulations the forces between particles are effectively screened by the simulation box. This helps to smooth the small fluctuations of forces arising with the periodic boundary conditions. It doesn't affect the mechanisms controlling the particle motion in the simulation box.

Second the collisional ionization / recombination process implemented in the code allows a fast joint equilibration of the electron population with the ionic charge state population. At the beginning ions and electrons are placed together in the simulation cell. This makes mandatory an equilibrium phase to take place first. This primitive evolution phase is driven by a fixed ion density and a constrained electron temperature. The equilibration has to be completed before any statistical analysis of the simulated 
plasma. The system evolution is considered as stationary, when the ionization events compensate the recombinations and the total energy fluctuations of the simulated particles remain small.

During the stationary evolution of the simulation cells, each ionization event of an ion starts with the placement of an electron at the ion. This placement means that the valence electron is in its fundamental state and has a vanishingly small kinetic energy. This position allows to know the instantaneous ionization potential $E_{i}$ of that ion. The ionization potential of ions with a given charge in the plasma is obtained as the average of the corresponding instantaneous ionization potentials and the IPD results from the difference with the ionization potential of the corresponding isolated ion.

Classical molecular dynamics is designed to solve ideal N-body problems. Here, ideal, means that the system under study relies on the simplest hypothesis. One of the main interest to use MD is to fully account for electron dynamics and for the structure of ion configurations. In the previous work reported, [10], the ideal system was defined as a stationary aluminum plasma at solid density and equal ion and electron temperature $T_{e}=T_{i}$. This choice is quite academic as we do not know if the generation of such a plasmas can be practically done. Another type of ideal system, composed with aluminum ions at room temperature, imbedded into hot electrons e.g., a few tens of eV, can be considered. This second ideal experiment corresponds to the conditions that could be obtained by a short radiation pulse on solid aluminum. In our MD simulation the only mechanism of temperature equilibration of two kinds of interacting particles e.g., ions and electrons, is a collisional mechanism. Coulomb explosion is not allowed as the system is infinite and there is no hot electron. The mass difference between aluminum ions and electrons ensures the coherence of the two temperature model because the necessary ion / electron equilibration phase can take place with a negligible heating of the ion component.

\section{Results and discussion}

Both the electrons and ions are responsible for the IPD, however, in the framework of our ion-electron MD simulations, their respective effect cannot be considered separately. With MD, the electron-ion interaction is not represented by a potential screened by the electrons themselves but accounts for all the surrounding electrons through binary interactions. Contrary to MD, theoretical models link the IPD to the electron plus ion Debye screening of ions with the average charge and the average ion-ion distance (SP model) or connect the IPD to the average static electric micro-field (EK model) generated by ions and electrons. Both these approaches based on an approximate ion effect, could provide some insight on the specific electron contribution to the IPD. In our model, electrons adjust dynamically to ions to compensate the ionic charge. Therefore, the electron plasma structure gives an approximate negative imprint of the ion structure with the difference that ions are practically motionless over a typical time of interest while electrons behave more or less like a fluid. A series of simulations with different 
temperature and ionic density will help the understanding of the main mechanisms giving rise to the IPD.

The difference between the two plasmas appears in the ion-ion pair correlation function.

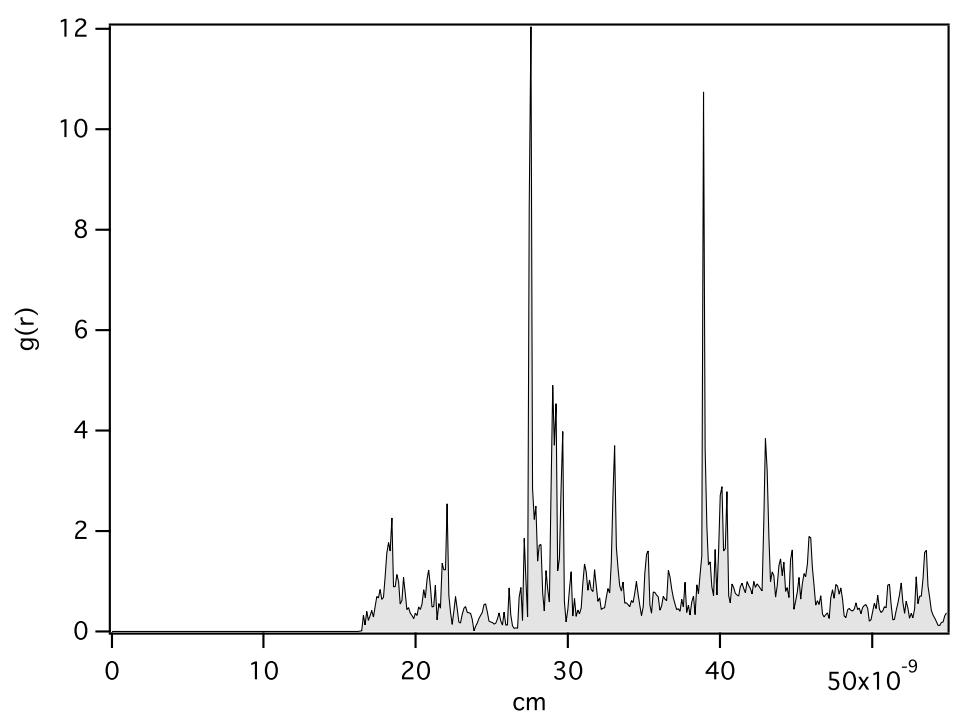

Figure 1. ion/ion pair correlation function at room temperature

For ions at room temperature a quasi crystalline ion configuration results into a series of peaks in the $g(r)$ plotted in fig. 1 while in fig. 2, at equal ion / electron temperature a smooth ordered structure is revealed only by a bump. The term "quasicrystalline" refers to our calculation process carried out on a set of independent initial

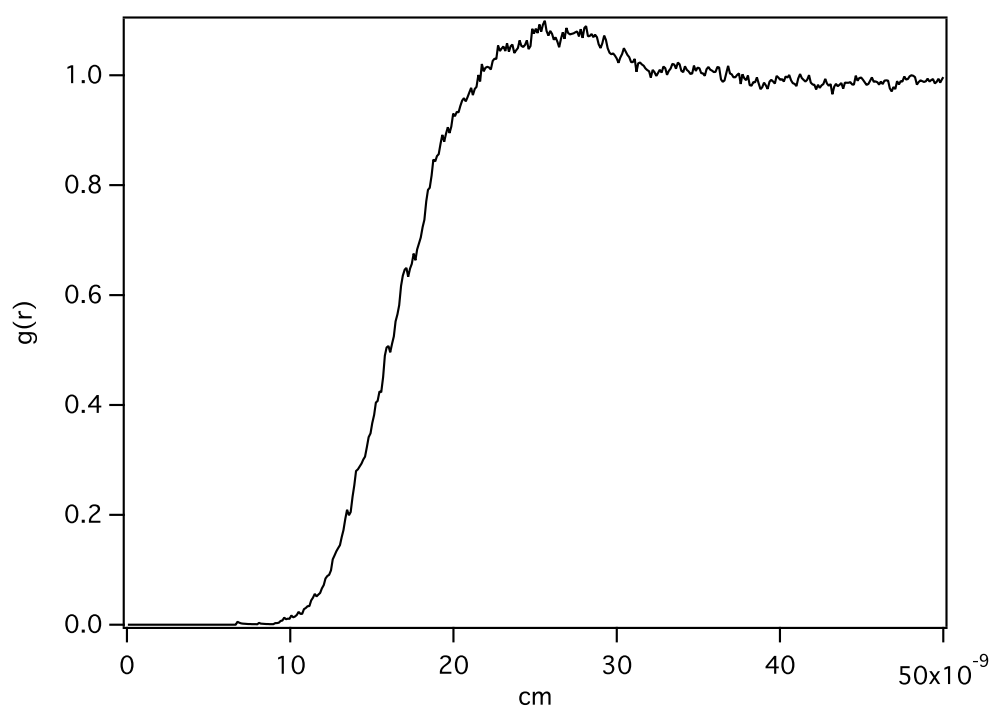

Figure 2. ion/ion pair correlation function at $T_{e}=T_{i}=50 \mathrm{eV}$ 
ion configurations at room temperature prepared at random but not at all like a perfect crystalline structure. Even though, the pair correlation function in fig. 1 is fairly noisy, this does not affect the IPD measurements.

The influence of the ionic structure on the IPD is shown in fig. 3 together with the comparison with the different models. Simulations show that there is an increase of the IPD for the case with ions at room temperature. An interpretation of this result associates the overlapping of the ion wells which depends on their short distance ordering to an increase of the IPD. At room temperature the quasi crystalline ordering results into a lowering of the energy required to free an electron from the ion configurations. Comparisons with theoretical models show that our simulation results fall in between both models.

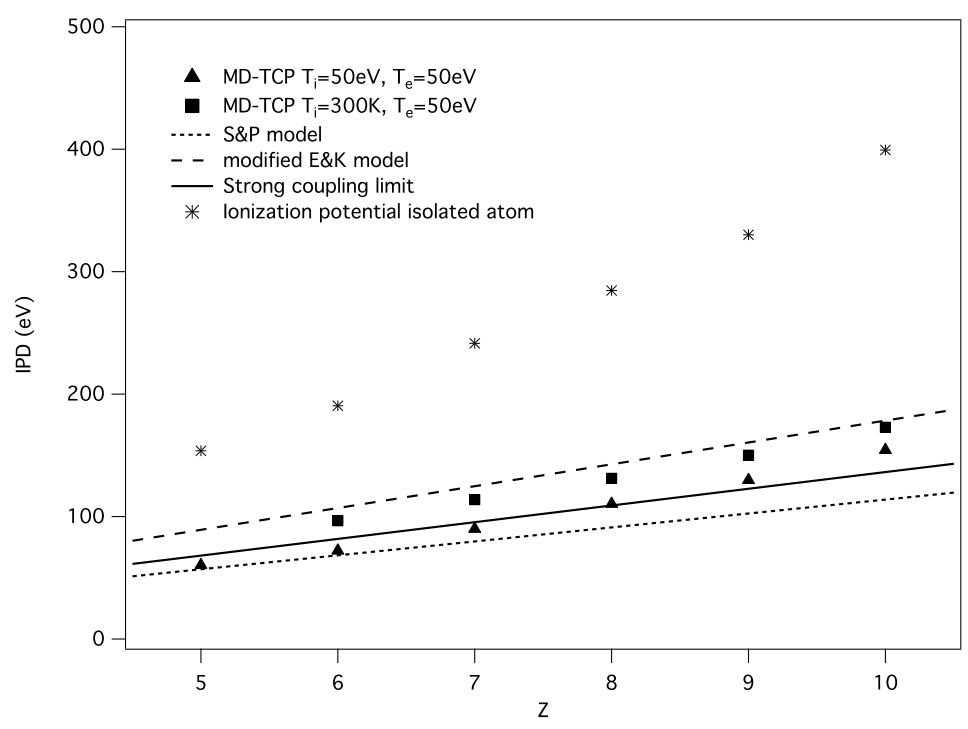

Figure 3. Influence of the ionic structure on the ionization potential depression (IPD).

The ion well overlapping mechanism which strongly depends on the ionic structure appears to be fundamental in the IPD formation. A compression of the plasma at a ionic density greater than solid density, $n_{i}=8 \times 10^{22} \mathrm{~cm}^{-3}$, increases this overlapping inducing an increase of the IPD as can be shown in fig. 4 .

Comparisons with theoretical models show here again that our simulation results fall in between both models and that the density variation is qualitatively well reproduced by both models even though our simulations predict an IPD increase twice less large than the theoretical models. Analyzing fig. 3 and 4, it appears that the IPD corresponding to a simulated system with ions at room temperature would be better represented by the EK model.

This model describes the lowering of the ionization potential as being due to the presence of an electric microfield and the ionization potential is defined as the difference between the ground state energy of the ion of charge $Z$ and that of the ion of charge $Z+1$. The IPD EK-model only depends on the total density (ion plus electron) and 


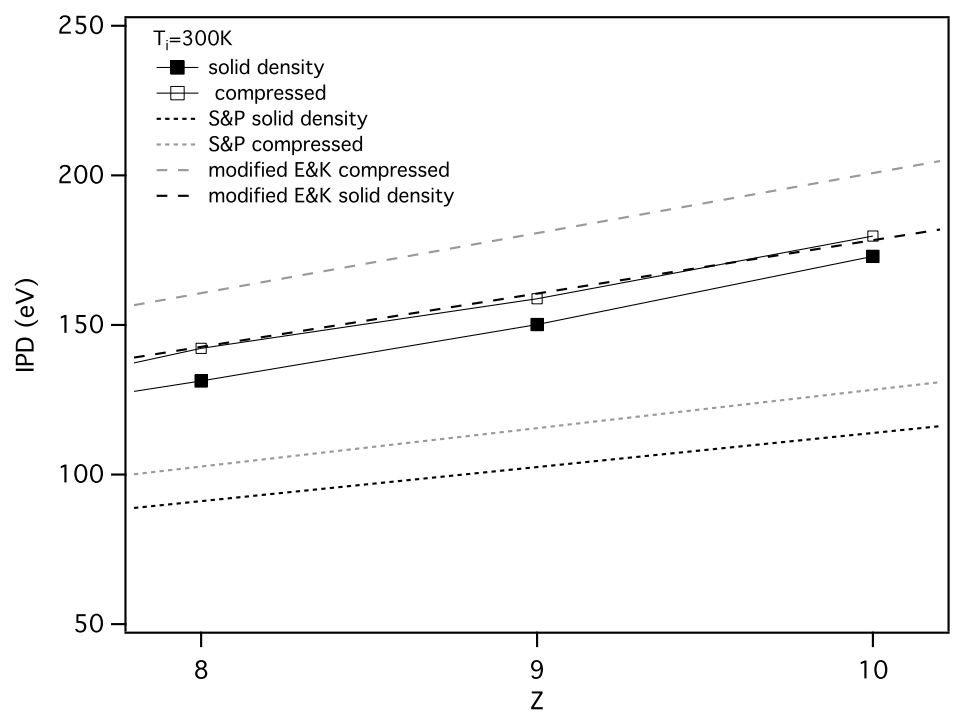

Figure 4. IPD simulation and theoretical model results for $T_{e}=50 \mathrm{eV}$. empty squares: compressed; black squares: solid density.

thus depends on the ionization amount. In our simulation model, the ionization amount is deduced from the total energy distribution of the electrons associated to the total number of electrons in the simulation box. For $T_{e}=50 \mathrm{eV}$, we have obtained $\bar{Z}=7.09$ for the ions at room temperature and $\bar{Z}=6.56$ at equilibrium. These results are consistent with the IPD increase at room temperature. We recall that in our model, the ionization / recombination process is a pure collisional process. In order to compare, our results with the models for different ionization amounts, a calculation at $T_{e}=160 \mathrm{eV}$ has been performed in both cases $T_{i}=300 \mathrm{~K}$ and $T_{i}=160 \mathrm{eV}$ corresponding to $\bar{Z}=9.55$ and $\bar{Z}=8.99$, respectively. The results are presented in fig. 5 .

When the electron temperature increases to $T_{e}=160 \mathrm{eV}$ two points have to be considered. First, the plasma composition is modified as confirmed by the increasing $\bar{Z}$. $Z=9$ and $Z=10$ are the only data available through sampling of the IPD. For simplicity, the helium-like and hydrogen-like charge state are not considered in our model. The corresponding ionization energies are greater than $2000 \mathrm{eV}$, i.e., more than one order of magnitude than the electron energy. A population of ions reduced to the highest charges has certainly a consequence on the overlapping of the ion wells. However, it should be noted that there is no practical way to determine the ion charge distribution because there is no mean to neatly separate an excited ion, i.e., an ion with a trapped electron, from a bare ion of the same charge. One can only assert that bare ions with $Z<9$ are almost absent with $T_{e}=160 \mathrm{eV}$. Second, the negative image of the ion structure given by the electrons is blurred in comparison with the case at $T_{e}=50 \mathrm{eV}$. The resulting effect is a net decrease of the IPD as shown in fig. 5. In the same figure one can see that at the highest temperature the increase of the IPD for ions at room temperature is larger than for $T_{e}=50 \mathrm{eV}$. A tentative interpretation 


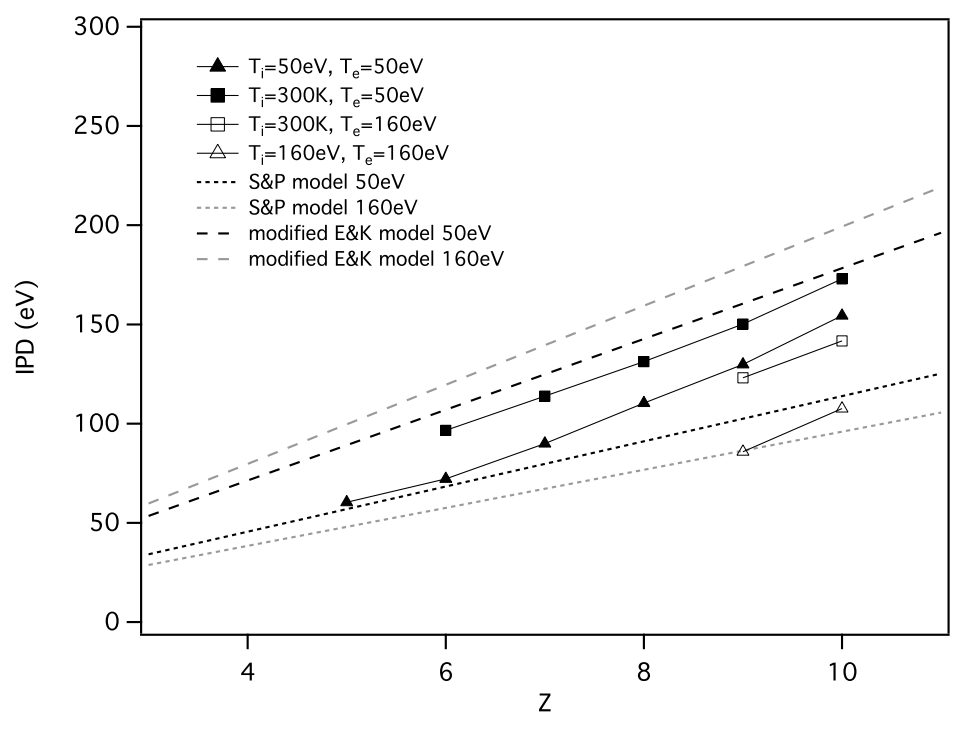

Figure 5. Comparisons of IPD simulation results with models for two different electronic temperatures.

of the effect due to faster electrons seems to be a reduced influence of the electrons on ions or in terms of electron screening present in the theoretical models, an increasing of the Debye length $\lambda_{D}$. According to this, it can be noticed that the variation with the temperature is better represented by the SP model than the EK model in which the temperature increase only appears in the value of the $\bar{Z}$.

The electron contributions to the IPD can be analyzed in terms of the electron total energy distributions.

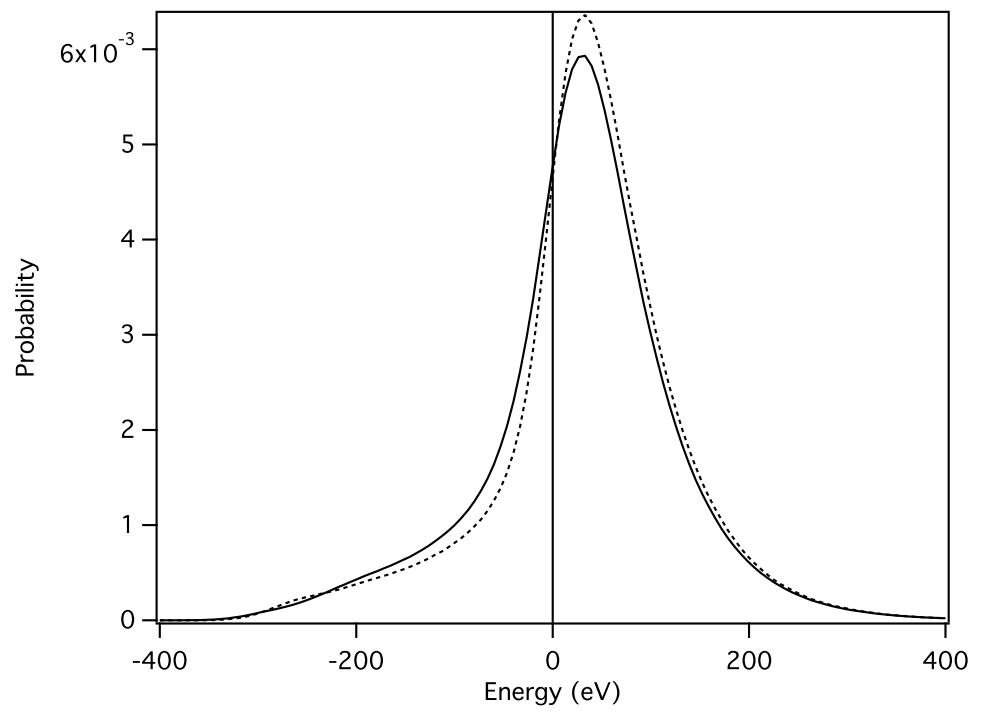

Figure 6. Electron total energy spectra. Continuous line: $T_{i}=T_{e}=50 \mathrm{eV}$, dotted line: $T_{i}=300 K$ 
These distributions roughly measure the population of trapped electrons with negative energy versus the free electron population with positive energy. They allow to estimate the free electron population and therefore the average ion charge $\bar{Z}$. In fig. 6 , the energy distributions for the two kinds of plasmas are slightly different. The transfer of a small amount of negative energy electron population to the positive energy region confirms that the ionization is made easier for the plasma with cold ions.

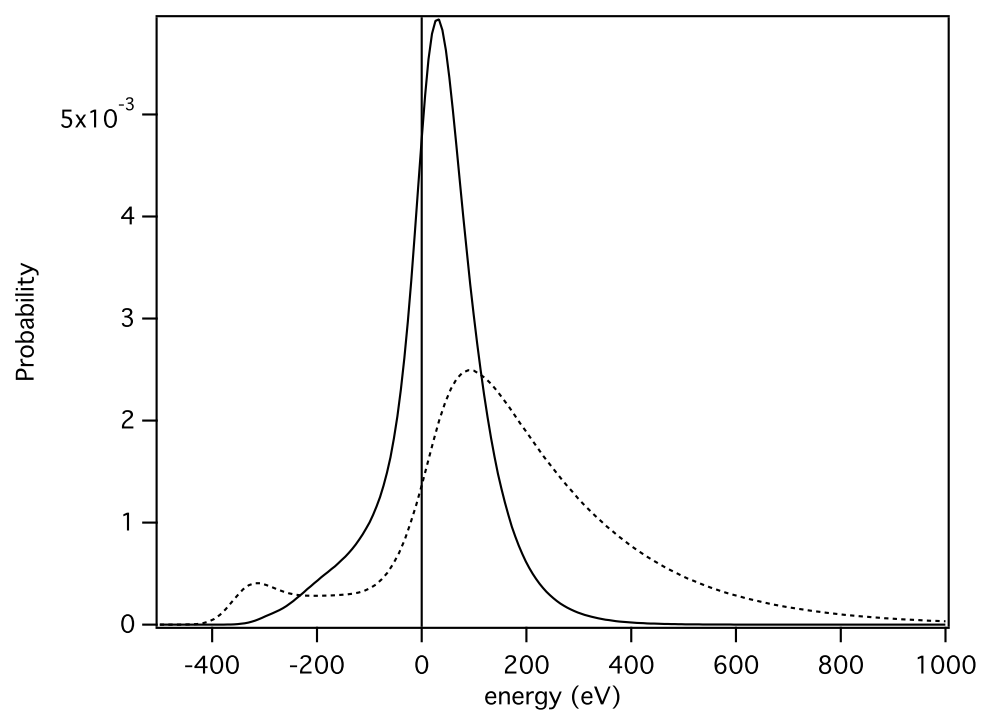

Figure 7. Electron total energy spectra. Continuous line: $T_{i}=T_{e}=50 \mathrm{eV}$, dotted line: $T_{i}=T_{e}=160 \mathrm{eV}$

In fig. 7, the electron total energy distribution functions are plotted for both equilibrium cases, $T_{i}=T_{e}=50 \mathrm{eV}$ and $T_{i}=T_{e}=160 \mathrm{eV}$. The bump in the negative energy region means that there is a small population of electrons deeply trapped around the highest charge state present at $T_{e}=160 \mathrm{eV}$ around $-350 \mathrm{eV}$. Within our approach these electrons are the classical equivalent of excited states below ionization occurring for ions with a net charge $Z-1$. The proportion of positive energy electrons is neatly increased at $160 \mathrm{eV}$ compared to $50 \mathrm{eV}$. At the same time, the total electron number in the simulation box itself is also increased in relation with the increase of the mean charge $\bar{Z}$.

Finally in fig. 8, we compare our simulation results with experimental data [3]. Even though considering ions at room temperature improves greatly the comparisons with experiment, some discrepancies remains. In the experiment, the ionization process is dominated by photoionization followed by $K L L$ Auger decay, so, it will only take place in a given ion if the photon energy of the pump lies above that ion's $K$ edge. As a consequence, each experimental point in fig. 8, corresponds to a different pump photon energy and thus to different plasma conditions. In our simulation models, the ionization process is a pure collisional process and each series of points is the result of a unique numerical experiment (all values of the IPD are measured in the same simulated 
plasma).

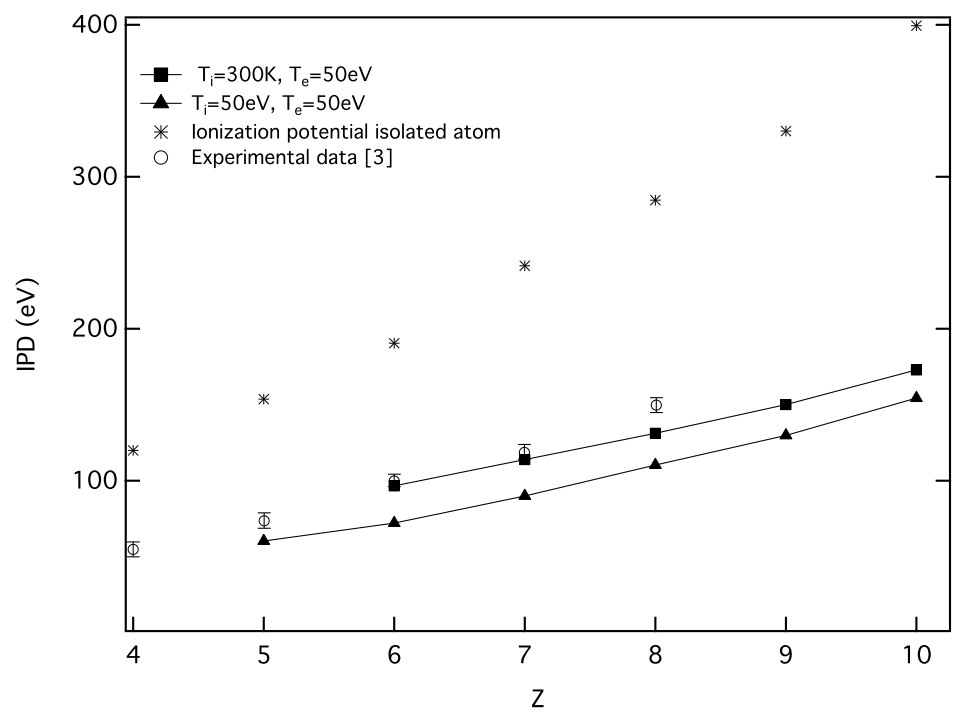

Figure 8. Comparisons with experimental data [3].

\section{Conclusion}

In this paper, a classical molecular dynamics simulation code, BINGO-TCP, has been used to infer the ionization potential depression in dense plasma at and out of equilibrium accounting for the influence of the free electrons and neighboring ions. Thanks to a ionization / recombination process associated to an ad-hoc ion-electron regularized potential, this code developed a few years ago, allows us to simulate neutral systems involving different charge state ions and electrons.

Calculations have been performed at two ionic densities, $n_{i}=6 \times 10^{22} \mathrm{~cm}^{-3}$ and $n_{i}=8 \times 10^{22} \mathrm{~cm}^{-3}$ and two different electronic temperatures, $T_{e}=50 \mathrm{eV}$ and $T_{e}=160 \mathrm{eV}$. Two types of plasmas have been considered, at equilibrium with $T_{i}=T_{e}$ and out of equilibrium with $T_{i}=300 \mathrm{~K}$.

Our conclusion about the IPD inside an aluminum plasma at solid density reflects the complexity of the problem. For some aspects our study should be considered as preliminary. Our simulations tend to show that the IPD depends not only on the average ion charge but on the relative placement of the ions. This attributes an important role to the ion temperature and suggests that overlapping of the ion wells is a major mechanism in the calculation of the IPD in plasmas.

An electron temperature change induces both a change in the electron adaptation to the ions and a change in the ion charges present in the plasma which in turn plays a role in the ion well overlapping mechanism.

Comparisons with the theoretical models, show that neither Stewart and Pyatt nor the modified Ecker and Kröll (with $C$ taken equals to 1 ) models are able to reproduced 
simulations results even though the modified EK model seems to be in better agreement with our results for ions at room temperature. The EK model has been developed for strong coupling plasmas and the SP model provides an answer to the IPD which yields the ion-sphere and the Debye-Hückel results as approximate limits. None of them had been designed to be used in non-equilibrium conditions. As a consequence, nothing in this study allows us to conclude that one or the other of the two models is better or not.

Even though, the simulated plasmas cannot be considered as being exactly in the same conditions as the experimental plasmas, the ionization process being purely collisional, comparisons with experimental data show an overall good agreement.

The model proposed in this work appears as an important tool to provide data for further discussion on IPD models.

\section{references}

[1] Stewart J C and Pyatt K D 1966 Astrophys. J. 1441203.

[2] Ecker G and Kröll W 1963 Phys. Fluids 662.

[3] Ciricosta O et al. 2012 Phys. Rev. Lett. 105065002.

[4] Hoarty D J et al. 2013 Phys. Rev. Lett. 110265003.

[5] Vinko S M et al. 2014 Nat. Commun. 53533.

[6] Crowley B J B 2014 HEDP 13 84-102.

[7] Iglesias C A 2014 HEDP 12 5-11.

[8] Son S K et al. 2014 Phys. Rev. X 40311004.

[9] Fletcher L B et al. 2014 Phys. Rev. Lett. 112145004.

[10] Calisti A, Ferri S and Talin B 2015 CPP DOI 10.1002/ctpp.201400087

[11] Vinko S et al. 2012 Nature 482 59-62

[12] Calisti A and Talin B 2011 CPP 51 vol 6 524-8

[13] Calisti A et al. 2009 HEDP 5307. 